\title{
ORIGINAL
}

\section{ESTIMACIÓN DE LA MORTALIDAD ATRIBUIBLE A ENFERMEDADES LABORALES EN ESPAÑA, 2004 (*)}

\author{
Ana M. García García (1,2), Rafael Gadea Merino (2) y Vicente López Martínez (2) \\ (1) Departamento de Medicina Preventiva y Salud Pública. Universidad de Valencia. \\ (2) Instituto Sindical de Trabajo, Ambiente y Salud. Valencia. \\ (*) Trabajo financiado con una ayuda del Ministerio de Trabajo y Asuntos Sociales (referencia FIPROS 2005-10).
}

\section{RESUMEN}

Fundamento: El registro de enfermedades profesionales en España resulta insuficiente para valorar el impacto de las enfermedades de origen laboral en nuestro país. En el presente estudio se estima la mortalidad por enfermedades laborales y sus costes en base a riesgos atribuibles estimados en la literatura internacional.

Métodos: Se aplican estimaciones de riesgo atribuible para la mortalidad de origen laboral calculadas por Nurminen y Karjalainen (Finlandia, 2001) al total de muertes por las enfermedades y grupos de edad relevantes en España y por comunidades autónomas en 2004. Se calculan los años potenciales de vida y de vida laboral perdidos. A partir de estos últimos, se estiman los costes actualizados de la pérdida de vida productiva debida a las muertes por enfermedades laborales en España.

Resultados: Según nuestras estimaciones, en 2004 se habrían producido en España cerca de 16.000 muertes por enfermedades relacionadas con exposiciones laborales, la mayoría en hombres $(87 \%)$. Estas muertes habrían causado cerca de 152.000 años potenciales de vida perdidos y algo más de 47.000 años potenciales de vida laboral perdidos, lo que conllevaría un coste actualizado en pérdidas de productividad entre 580 y 1.000 millones de euros.

Conclusiones: En 2004 el registro de enfermedades profesionales en España sólo recogió dos muertes por esta causa. Sin embargo, los resultados del presente estudio ponen de manifiesto la importancia de las condiciones de trabajo como determinantes de muertes evitables en la población y la inminente necesidad de sistemas de vigilancia y prevención adecuados para afrontar en toda su magnitud este problema para la salud pública.

Palabras clave: Salud laboral. Enfermedades laborales. Fracción atribuible. Esperanza de vida. Costes.

Correspondencia:

Ana M. García

Instituto Sindical de Trabajo, Ambiente y Salud

Almirante, 3, 4 4.46003 Valencia

Correo-e: anagar@uv.es

\section{ABSTRACT \\ Estimate of the Mortality \\ Rate Attributable \\ to Occupational Diseases in Spain, 2004}

Background: Occupational diseases are not registered in Spain to a sufficient degree so as to be able to evaluate the impact thereof in our country. In this study, the occupational disease-related mortality and the costs thereof are estimated based on attributable risks calculated in international literature.

Methods: Attributable risk estimates are applied for the occupationally-caused deaths rate calculated by Nurminen and Karjalainen (Finland, 2001) are applied to the total number of deaths by the relevant diseases and age groups in Spain and in Autonomous Communities in 2004. The potential years of life and working life lost are calculated. Based upon the years of working life lost, the updated costs of the loss of productive life due to deaths caused by occupational diseases in Spain are estimated.

Results: According to our estimates, nearly 16,000 deaths would have occurred in Spain due to occupational exposurerelated diseases, the majority in males $(87 \%)$. These deaths could have caused nearly 152,000 potential years of life lost and somewhat over 47,000 potential years of working life lost, which would entail an updated cost in productivity losses of 580 million - 1 billion euros.

Conclusions: In 2004, the occupational disease register in Spain included solely two deaths due to this cause. The results of this study however reveal the importance of working conditions as determining factors in preventable deaths among the population and the imminent need for adequate surveillance and prevention systems for dealing with this public health problem in its full magnitude.

Key words: Occupational health. Occupational diseases. Attributable fraction. Life expectancy. Costs. 


\section{INTRODUCCIÓN}

Los factores de riesgo presentes en el lugar de trabajo son causa frecuente de morbilidad y mortalidad en la población. Se ha calculado que el $16 \%$ de los procesos atendidos en centros de Atención Primaria de Salud están probablemente relacionados con las condiciones de trabajo ${ }^{1}$. Las redes de médicos centinela de salud laboral, como la impulsada por el Instituto Navarro de Salud Laboral, han demostrado la frecuencia con la que patologías de origen laboral (tales como tendinitis del miembro superior, síndrome del túnel carpiano, asma o dermatosis) son atendidas por el sistema sanitario público sin llegar a ser reconocidas como enfermedades profesionales $^{2}$. Por otra parte, según estimaciones disponibles, en España un 25\% de los trabajadores (algo más de 5 millones de personas en 2004) estarían expuestos a cancerígenos en el lugar de trabajo ${ }^{3,4}$.

El registro de enfermedades profesionales en España es el único sistema oficial de vigilancia de patologías de origen laboral a nivel nacional. Según la normativa vigente hasta 2006, las empresas tenían la obligación de declarar todos los casos de enfermedad profesional que ocurrieran en los trabajadores. Sin embargo, las limitaciones de este sistema como herramienta para la vigilancia y prevención de los riesgos para la salud laboral son ampliamente reconocidas 5 . En primer lugar, en el registro oficial sólo se recogen las enfermedades incluidas en el Cuadro de Enfermedades Profesionales (Real Decreto 1995/1978, derogado y actualizado recientemente por el Real Decreto 1299/2006), un listado manifiestamente incompleto. Tanto en su versión de 1978 como en la lista recientemente actualizada no se contemplan, por ejemplo, trastornos mentales de origen labo$\mathrm{ral}^{6}$. Otros procesos como los tumores malignos, las enfermedades cardiovasculares o las enfermedades respiratorias de origen laboral quedan pobremente reflejados en el registro de enfermedades profesionales. De hecho, el mayor número de enfermedades profesiona- les reconocidas en España son trastornos osteomusculares (71\%), cutáneos (13\%) o neurológicos $(12 \%)$ de naturaleza leve ${ }^{7}$.

La mortalidad por enfermedades laborales es un valioso indicador del estado de la salud laboral en un país. Según estimaciones llevadas a cabo en diferentes contextos, el total de muertes producidas por enfermedades laborales podría ser entre 5 y 20 veces mayor que la mortalidad por accidentes de trabajo ${ }^{8}$, otro problema de salud en los trabajadores generalmente mejor conocido y reconocido por las estadísticas oficiales. Sin embargo, en España, frente a las cerca de 1.000 muertes por accidente de trabajo registradas anualmente sólo se reconocieron entre 0 y 3 muertes anuales por enfermedad profesional entre 2000 y $2005^{9}$.

En la literatura internacional existen estudios que estiman el impacto de la mortalidad laboral en diferentes países. En general estos estudios se basan en el cálculo de los riesgos atribuibles de mortalidad para diferentes exposiciones laborales estimados en base a los riesgos relativos de muerte por dichas exposiciones y la prevalencia de exposición a los correspondientes factores de riesgo para las poblaciones de interés. Las estimaciones más exhaustivas y recientes de mortalidad atribuible a exposiciones laborales se encuentran en los estudios de Nurminen y Karjalainen $(2001)^{10}$ y Steenland y cols. $(2003)^{11}$, para población finlandesa y estadounidense respectivamente.

Nurminen y Karjalainen ${ }^{10}$ basan sus estimaciones en una revisión exhaustiva de la literatura epidemiológica y la información relativa a la prevalencia de exposiciones laborales en los trabajadores finlandeses según una matriz empleo-exposición previamente elaborada en Finlandia. Para sus estimaciones consideraron los rangos de edad relevantes para cada enfermedad en función de la edad media de jubilación en Finlandia (59 años) y los periodos de latencia de los procesos incluidos. Por su parte, Steenland y cols. ${ }^{11}$ publican las 
estimaciones nacionales más recientes para Estados Unidos según cálculos propios basados en una revisión de la bibliografía científica. Las estimaciones de este último estudio son en general similares a las del trabajo de Nurminen y Karjalainen, aunque no incluyen información suficientemente detallada por sexos y excluyen algunas causas de mortalidad incluidas en el trabajo de los finlandeses.

Hace algunos años llevamos a cabo una estimación del impacto de las enfermedades laborales en España en 1999 en términos de mortalidad, incidencia y prevalencia $^{12}$. El objetivo del presente trabajo, actualizando y ampliando el citado estudio previo, ha sido estimar la mortalidad por enfermedades laborales en España en 2004, tanto para el conjunto nacional como por comunidades autónomas, para ambos sexos, incluyendo una valoración de los costes de la mortalidad laboral en base a los años de vida laboral perdidos.

\section{MATERIAL Y MÉTODOS}

\section{Estimaciones de mortalidad laboral en España}

En el presente trabajo hemos aplicado las estimaciones de riesgo atribuible por grupos de edad calculadas en el estudio de Nurminen y Karjalainen ${ }^{10}$ (tabla 1) por parecernos las más completas y rigurosas, además de ser las únicas disponibles por sexos.

Para obtener el número de muertes de origen laboral en 2004 en España, por comunidades autónomas, totales y por sexos, se han aplicado los riesgos atribuibles de la tabla 1 al total de muertes en la población por el correspondiente grupo de enfermedades y en los grupos de edad relevantes. La mortalidad poblacional se obtiene de los datos de defunciones según la causa de muerte del Instituto Nacional de Estadística (INE) en el año $2004^{13}$. Tanto en el estudio finlandés como en los datos del INE los grupos de enfermedades se conforman según la Clasificación Internacional de Enfermedades en su décima revisión (CIE-10). Sin embargo, en el estudio de Nurminen y Karjalainen se utiliza la lista completa, mientras que para aplicar los riesgos atribuibles a los datos del INE se ha utilizado la lista reducida de esta clasificación por ser la única disponible a nivel de comunidad autónoma.

\section{Años potenciales de vida perdidos}

Para estimar el número de años potenciales de vida perdidos (APVP) por muertes

Tabla 1

Riesgos atribuibles proporcionales para grupos principales de enfermedades laborales ( $\%$ de muertes debidas a exposiciones laborales en cada grupo)

estimados en el estudio de Nurminen y Karjalainen ${ }^{10}$

\begin{tabular}{|l|c|c|c|c|}
\hline & CIE-10 $^{\mathbf{a}}$ & Total $^{\mathbf{b}}$ & Hombres $^{\mathbf{b}}$ & Mujeres $^{\mathbf{b}}$ \\
\hline Enfermedades infecciosas & A00-B99 & 8,8 & 4,8 & 32,5 \\
\hline Tumores & C00-C97 & 8,4 & 13,8 & 2,2 \\
\hline Trastornos mentales & F00-F99 & 3,5 & 7,3 & 1,8 \\
\hline Enfermedades del sistema nervioso & G00-H95) & 3,1 & 5,1 & 1,7 \\
\hline Enfermedades del aparato circulatorio & I00-I99 & 12,4 & 14,4 & 6,7 \\
\hline Enfermedades del aparato respiratorio & J00-J99 & 4,1 & 6,8 & 1,1 \\
\hline Enfermedades del aparato digestivo & K00-K93 & 2,1 & 2,3 & 1,5 \\
\hline Enfermedades del sistema genitourinario & N00-N99 & 1,3 & 3,0 & 0,4 \\
\hline Total & & $\mathbf{6 , 7}$ & $\mathbf{1 0 , 2}$ & $\mathbf{2 , 1}$ \\
\hline
\end{tabular}

${ }^{a}$ Categorías de enfermedad según CIE-10 (lista detallada).

${ }^{\mathrm{b}}$ Los riesgos atribuibles se estiman para muertes ocurridas en población de 25 años y más excepto para enfermedades cardiovasculares (población entre 25-74 años) y enfermedades digestivas e infecciosas (población entre 25-64 años). 
laborales se ha calculado el porcentaje de muertes para cada grupo de enfermedad por grupos de edad (quinquenales) en los tramos de edad considerados por Nurminen y Karjalainen ${ }^{10}$. Los APVP se han estimado conjuntamente para ambos sexos. A partir de dichas proporciones se obtiene el total de muertes estimadas para cada grupo de enfermedades y en cada tramo de edad. Este número se multiplica por la diferencia entre el punto medio del intervalo del grupo de edad correspondiente y la esperanza de vida al nacer (79 años, media para ambos sexos en $2004^{14}$ ). Finalmente se obtiene el total de años de vida perdidos para cada grupo de enfermedad y para el conjunto de ellas.

\section{Costes por años de vida laboral perdidos}

Se han calculado los costes económicos indirectos de las muertes laborales en términos de costes monetarios derivados de la productividad laboral perdida según los años potenciales de vida laboral perdidos (APVLP). El método empleado utiliza como hipótesis básica la equivalencia entre el valor de la producción perdida y el salario asociado a dicha producción. Es decir, un día de pérdida de trabajo implica una pérdida de la producción igual al salario que se percibiría por ese día.

En primer lugar se calcula el número de años de trabajo perdidos (APVLP) a partir de la edad media a la que se produce la muerte (obtenida de igual manera que para el cálculo de APVP, ver apartado anterior) y la edad media de jubilación (65 años). La cuantificación monetaria de los APVLP se realiza a partir de la ganancia salarial media $^{15}$. El valor actual de la ganancia salarial perdida por los APVLP depende de la tasa de crecimiento acumulada del porcentaje de aumento de la productividad y de la tasa de descuento que apliquemos, que suele venir determinada por el tipo de interés de mercado. Dicha tasa de descuento, en nuestro caso, se ha obtenido a partir del tipo oficial del Banco Central Europeo ${ }^{16}$. Como escenario más probable hemos establecido una tasa de descuento del $3 \%$ y un crecimiento de la productividad del $1 \%$. De hecho, para el año 2006 el euribor presenta una media próxima al 3\% (2,6\% en el primer trimestre y $3,2 \%$ en el tercer trimestre), habiéndose situado el crecimiento de la productividad en los últimos años en torno al medio punto ${ }^{16}$. Para el análisis de sensibilidad se aplican tasas alternativas sobre este escenario. Concretamente, consideramos un crecimiento de la tasa de productividad de $0 \%, 1 \%$ y $2 \%$ y un crecimiento de la tasa acumulativa de descuento de $1 \%, 3 \%$ y $5 \%$. El escenario más pesimista, es decir el que supone una valoración de la pérdida potencial de producción mayor, vendrá dado por la mayor tasa de crecimiento de la productividad (2\%) y la menor tasa aplicada de descuento (1\%). El escenario que presenta un coste menor será aquél que viene determinado por una menor tasa de crecimiento de la productividad $(0 \%)$ y una mayor tasa de descuento (5\%).

Así, se multiplicará el número de muertes ocurridas en cada tramo de edad por la ganancia salarial media actualizada en base al crecimiento de la productividad previsto y la tasa de descuento aplicada. La formulación matemática es la que se utiliza para el cálculo del valor actual de rentas variables que evolucionan temporalmente en progresión geométrica ${ }^{17}$.

\section{RESULTADOS}

La tabla 2 presenta las estimaciones del número de muertes atribuibles a exposiciones laborales para el conjunto nacional en el año 2004. Según las estimaciones realizadas, en 2004 habrían fallecido en España 16.125 personas como consecuencia de exposiciones relacionadas con su trabajo. Esta mortalidad afectaría principalmente a hombres ( $87 \%$ del total). El mayor número 
Tabla 2

Estimación de las muertes por enfermedades laborales según tipos principales de causas. España, 2004

\begin{tabular}{|c|c|c|c|c|c|}
\hline & \multirow{2}{*}{ CIE-10 ${ }^{\mathrm{a}}$} & \multirow{2}{*}{ Total muertes ${ }^{\mathbf{b}}$} & \multirow{2}{*}{$\mathbf{R A}(\%)^{\mathrm{c}}$} & \multicolumn{2}{|c|}{ Muertes por exposiciones laborales ${ }^{d}$} \\
\hline & & & & $\mathbf{N}$ & $\%$ \\
\hline \multicolumn{6}{|l|}{ HOMBRES } \\
\hline Infecciosas y parasitarias & $001-008$ & 1.895 & 4,8 & 91 & 0,6 \\
\hline Tumores & 009-041 & 62.659 & 13,8 & 8647 & 61,7 \\
\hline Trastornos mentales & $046-049$ & 4.054 & 7,3 & 296 & 2,1 \\
\hline Sistema nervioso & $050-052$ & 5.503 & 5,1 & 281 & 2,0 \\
\hline Aparato circulatorio & 053-061 & 20.403 & 14,4 & 2938 & 21,0 \\
\hline Aparato respiratorio & $062-067$ & 23.101 & 6,8 & 1571 & 11,2 \\
\hline Aparato digestivo & $068-072$ & 3.050 & 2,3 & 70 & 0,5 \\
\hline Sistema genitourinario & $077-080$ & 4.051 & 3 & 122 & 0,9 \\
\hline Total & & & & 14.016 & 100 \\
\hline \multicolumn{6}{|l|}{ MUJERES } \\
\hline Infecciosas y parasitarias & $001-008$ & 569 & 32,5 & 185 & 8,8 \\
\hline Tumores & 009-041 & 37.367 & 2,2 & 822 & 39,0 \\
\hline Trastornos mentales & 046-049 & 7.809 & 1,8 & 141 & 6,7 \\
\hline Sistema nervioso & $050-052$ & 8.378 & 1,7 & 142 & 6,7 \\
\hline Aparato circulatorio & 053-061 & 9.132 & 6,7 & 612 & 29,0 \\
\hline Aparato respiratorio & $062-067$ & 15.919 & 1,1 & 175 & 8,3 \\
\hline Aparato digestivo & $068-072$ & 901 & 1,5 & 14 & 0,7 \\
\hline Sistema genitourinario & $077-080$ & 4.475 & 0,4 & 18 & 0,9 \\
\hline Total & & & & 2.109 & 100 \\
\hline
\end{tabular}

a Categorías de enfermedad según CIE-10 (lista reducida).

b Total de muertes (España, 2004) en cada grupo para las siguientes categorías de edad: enfermedades infecciosas y enfermedades del aparato digestivo: 25-64 años; tumores, trastornos mentales, enfermedades del sistema nervioso, enfermedades del aparato respiratorio y enfermedades del sistema genitourinario: 25 años y más; enfermedades del aparato circulatorio: 25-74 años. Fuente: Instituto Nacional de Estadística, 2004.

c Riesgo atribuible: proporción (\%) de muertes relacionadas con exposiciones laborales para cada grupo de causas y categorías de edad consideradas. Fuente: Nurminen y Karjalainen, 2001 (ver Tabla 1).

${ }^{d}$ Número de muertes de origen laboral para cada grupo de causas y porcentaje de mortalidad laboral en cada grupo sobre el total de muertes laborales.

de muertes de origen laboral se debería a tumores malignos, seguidos por las enfermedades cardiovasculares. Como tercera causa de mortalidad de origen laboral en los hombres aparecen las enfermedades del aparato respiratorio y las enfermedades infecciosas y parasitarias en las mujeres. El total estimado de años potenciales de vida perdidos (APVP) a nivel nacional por las muertes de origen laboral en 2004 sería de 151.792 años, en su mayor parte (51\%) debidos a la mortalidad por cáncer laboral ( $\mathrm{n}=77.418$ años), seguida por las muertes cardiovasculares (36\%, 55.110 años) y las muertes por enfermedades infecciosas adquiridas en el trabajo (5\%, 7.413 años).

En la tabla 3 se presenta la estimación del total de muertes atribuibles al trabajo por comunidades autónomas y por sexo, así
Tabla 3

Estimación del número de muertes (por sexo y totales) y años potenciales de vida perdidos (totales) atribuibles a exposiciones laborales por comunidades autónomas, 2004

\begin{tabular}{|c|c|c|c|c|}
\hline & \multicolumn{3}{|c|}{ Muertes (n) } & \multirow{2}{*}{$\begin{array}{r}\text { APVP } \\
\text { (años) }\end{array}$} \\
\hline & Hombres & Mujeres & Total & \\
\hline Andalucía & 2.388 & 367 & 2.755 & 27.395 \\
\hline Aragón & 481 & 68 & 549 & 4.909 \\
\hline Asturias & 480 & 66 & 546 & 4.935 \\
\hline Baleares & 265 & 37 & 302 & 2.763 \\
\hline Canarias & 487 & 82 & 569 & 1.125 \\
\hline Cantabria & 200 & 28 & 228 & 1.998 \\
\hline Castilla-León & 984 & 130 & 1.114 & 9.669 \\
\hline Castilla-La Mancha & 615 & 94 & 709 & 6.375 \\
\hline Cataluña & 2.187 & 330 & 2.517 & 22.948 \\
\hline Comunidad Valenciana & 1.463 & 227 & 1.690 & 16.433 \\
\hline Extremadura & 389 & 57 & 446 & 4.194 \\
\hline Galicia & 1.065 & 155 & 1.220 & 11.170 \\
\hline Madrid & 1.496 & 239 & 1.735 & 16.544 \\
\hline Murcia & 354 & 55 & 409 & 3.914 \\
\hline Navarra & 186 & 26 & 212 & 1.894 \\
\hline País Vasco & 745 & 107 & 852 & 7.775 \\
\hline Rioja & 95 & 14 & 109 & 992 \\
\hline
\end{tabular}

a Años potenciales de vida perdidos. 
Tabla 4

Estimación del número de muertes por las tres causas principales de mortalidad (ambos sexos) atribuibles a exposiciones laborales por comunidades autónomas, 2004

\begin{tabular}{|l|c|c|c|}
\hline & Tumores malignos & Aparato circulatorio & Aparato respiratorio \\
\hline Andalucía & 1.524 & 733 & 292 \\
\hline Aragón & 338 & 101 & 63 \\
\hline Asturias & 327 & 109 & 60 \\
\hline Baleares & 182 & 62 & 30 \\
\hline Canarias & 319 & 158 & 26 \\
\hline Cantabria & 144 & 38 & 129 \\
\hline Castilla-León & 711 & 188 & 91 \\
\hline Castilla-La Mancha & 420 & 140 & 249 \\
\hline Cataluña & 1.521 & 486 & 170 \\
\hline Comunidad Valenciana & 942 & 424 & 51 \\
\hline Extremadura & 264 & 102 & 144 \\
\hline Galicia & 741 & 248 & 220 \\
\hline Madrid & 1.040 & 340 & 52 \\
\hline Murcia & 230 & 96 & 26 \\
\hline Navarra & 130 & 38 & 11 \\
\hline País Vasco & 525 & 171 & 20 \\
\hline Rioja & 69 & & \\
\hline
\end{tabular}

Tabla 5

Estimación de los costes derivados de los años de vida laboral perdidos por la mortalidad por enfermedades laborales según diferentes escenarios para la pérdida de productividad derivada. España, 2004

\begin{tabular}{|l|c|c|c|c|}
\hline & & Tasa de descuento & & \\
\hline & & $\mathbf{1 \%}$ & $\mathbf{3 \%}$ & $\mathbf{5 \%}$ \\
\hline Tasa de crecimiento de la productividad & $0 \%$ & $890.210 .949,36 €$ & $668.340 .769,96 €$ & $577.159 .290,79 €$ \\
\hline & $1 \%$ & $943.678 .251,00 €$ & $718.300 .221,14 €$ & $615.361 .795,18 €$ \\
\hline & $2 \%$ & $1.034 .829 .421,86 €$ & $775.009 .395,24 €$ & $658.250 .625,31 €$ \\
\hline
\end{tabular}

como el total de años potenciales de vida perdidos para ambos sexos en cada comunidad autónoma. En la tabla 4 se detalla el número estimado de muertes para las tres principales causas de mortalidad laboral (tumores malignos, enfermedades cardiovasculares y enfermedades respiratorias) por comunidades autónomas.

Por último, en la tabla 5 se presentan los costes indirectos de la mortalidad laboral debidos a las pérdidas de productividad por los años de vida laboral perdidos. Se ha estimado un total de 47.312 años potenciales de vida laboral perdidos por las muertes de origen laboral ocurridas en 2004, lo que en el escenario de menor coste (menor crecimiento de la productividad y mayor tasa de descuento) supondría costes cercanos a los 580 millones de euros. En el escenario de mayor coste (máximo crecimiento de productividad, mínima tasa de descuento) los costes superarían los 1.000 millones de euros.

\section{DISCUSIÓN}

Aplicando los riesgos atribuibles calculados por Nurminen y Karjalainen ${ }^{10}$ en población finlandesa para estimar la mortalidad de origen laboral, obtendríamos para España en el año 2004 un total de 16.000 muertes debidas a enfermedades contraídas como consecuencia de exposiciones en el lugar de trabajo. La mayoría de estas muertes $(87 \%)$ se producirían en hombres. En total, estas muertes causarían cerca de 
152.000 años potenciales de vida perdidos y algo más de 47.000 años potenciales de vida laboral perdidos, lo que conllevaría un coste actualizado en pérdidas de productividad entre 580 y 1.000 millones de euros.

Si relacionamos estas estimaciones con la mortalidad registrada por accidentes de trabajo a nivel nacional $(\mathrm{n}=1.459$ en $2004)^{18}$, la mortalidad por enfermedades laborales supondría el $92 \%$ del total de la mortalidad de origen laboral, quedando el $8 \%$ restante a la mortalidad por accidentes de trabajo. Esta relación sería bastante similar para los correspondientes resultados por comunidades autónomas (datos no mostrados). Por otra parte, el número total de APVP por las enfermedades laborales consideradas en este trabajo supondría el $17 \%$ del total de APVP producidos por estas mismas enfermedades en el conjunto de la población en el año 2001 según datos del Centro Nacional de Epidemiología ${ }^{19}$.

En 2004 el registro de enfermedades profesionales recogió 2 muertes por esta causa, ambas debidas a "carcinoma primitivo de bronquio o pulmón por asbesto" ". Los datos disponibles en países con un contexto similar al nuestro $8,10,11,20$ sugieren claramente que las cifras oficiales en España subestiman en gran medida el verdadero impacto de la mortalidad por exposiciones laborales. Sin embargo, el método aplicado en el presente trabajo no está exento de limitaciones.

Como ya se ha indicado, Nurminen y Karjalainen $^{10}$ estimaron los riesgos atribuibles para la mortalidad de origen laboral en base a una revisión exhaustiva de la evidencia epidemiológica al respecto y a la prevalencia de exposición a riesgos laborales según datos de una matriz empleo-exposición que incluye estimaciones de la frecuencia de exposición a 74 agentes laborales en población finlandesa (FINJEM). Al aplicar estos mismos riesgos atribuibles a la población española, por comunidades autónomas y por sexos, asumimos que la prelavencia de exposición a riesgos laborales en España es comparable a la de Finlandia. Pero esta asunción probablemente no se ajusta a la realidad. De hecho algunos datos disponibles señalan que los riesgos laborales en Finlandia son de menor magnitud que en nuestro país. Por ejemplo, según Eurostat ${ }^{21}$, las tasas de accidentes de trabajo mortales por 100.000 trabajadores, excluyendo los que se producen in itinere y por causas médicas y estandarizadas por sector económico, serían respectivamente para Finlandia y España de 1,9 por 100.000 y 3,7 por 100.000 , un indicador fiable que sugiere una mejor situación de la salud laboral en Finlandia en comparación con la de España. Por otra parte, la incidencia de las enfermedades profesionales en Finlandia muestra en los últimos años una clara tendencia descendente que se considera reflejo de la gradual mejora de las condiciones de trabajo en Finlandia durante los últimos años ${ }^{22}$. Por los datos que tenemos disponibles en España, bastante más limitados, no parece ser esta la tendencia en nuestro país en los últimos años ${ }^{23}$.

Aunque las condiciones de trabajo puedan ser más favorables en Finlandia en comparación con España, lo que determinaría que nuestras estimaciones estuvieran infravalorando la mortalidad atribuible a exposiciones laborales en España, se deben señalar sin embargo otras diferencias entre ambos países que pudieran tener efectos opuestos, o cuanto menos inciertos. Por ejemplo, también según Eurostat ${ }^{21}$, la proporción de mujeres ocupadas (en el grupo de edad de 15 a 64 años) en Finlandia en 2004 era del $65,6 \%$, frente al 48,3\% en ese mismo año para España. Por tanto, al extrapolar los estimadores obtenidos para población finlandesa estaríamos sobrevalorando la prevalencia de exposición de las mujeres españolas a riesgos laborales, y por tanto la mortalidad atribuible a estos riesgos en estas mujeres.

Otra fuente potencial de error en nuestras estimaciones tendría relación con la calidad 
de la evidencia epidemiológica utilizada en el cálculo de los riesgos atribuibles, tanto en términos de su disponibilidad como de su validez interna y externa. Aunque es mucho el conocimiento del que se dispone sobre los efectos de las exposiciones laborales sobre la salud de los trabajadores, todavía quedan muchas áreas sobre las que la información es insuficiente. Algunos efectos "clásicos", como el cáncer o determinadas enfermedades pulmonares, han recibido una gran parte de la atención de investigadores de todo el mundo, mientras que otros problemas, como alteraciones reproductivas, neurodegenerativas, digestivas, renales, vasculares, hormonales o del sistema inmune, son mucho peor conocidos y quedan con frecuencia excluidos de las distintas fuentes de estimación. Del mismo modo, suele haber más información epidemiológica disponible sobre los efectos de exposiciones laborales "clásicas", tales como determinados contaminantes químicos o físicos, que sobre exposiciones relacionadas con la organización del trabajo y de las tareas, de naturaleza ergonómica o psicosocial. Otro elemento sobre el que existe escaso conocimiento es el efecto interactivo de las distintas exposiciones. Habitualmente se considera el riesgo asociado con la exposición a un determinado factor, pero no se dispone apenas de información sobre el efecto de la exposición simultánea a múltiples factores, siendo ésta última la situación habitual en los lugares de trabajo.

Cabe también señalar que se dispone de menor información referida a determinados colectivos de trabajadores y situaciones de exposición laboral. De hecho una mayoría de la información epidemiológica existente se basa en estudios realizados en empresas grandes y sobre colectivos predominantemente masculinos. La información descriptiva sobre las situaciones que afectan a las empresas pequeñas o a las mujeres trabajadoras $^{24}$ es mucho menos frecuente, pudiendo concurrir en estos casos condiciones de exposición más extremas (mayor carga ergonómica o psicosocial en mujeres, peores condiciones de protección y prevención en las empresas más pequeñas). Las condiciones de los trabajadores autónomos y colectivos especiales como los inmigrantes ${ }^{25}$, también potencialmente peores, escapan igualmente de nuestro conocimiento y suelen quedar excluidas de los cálculos realizados en estudios como el de Nurminen y Karjalainen y otros ${ }^{10,11,20}$.

Por otra parte, el registro de la causa de muerte habitualmente se basa en la causa principal del fallecimiento. Sin embargo, en la muerte influyen tanto causas principales como secundarias. El impacto de las exposiciones laborales sobre estas causas secundarias o como coadyuvantes de la mortalidad queda habitualmente excluido en las estimaciones.

En el sentido opuesto, una fuente de error que llevaría a la sobrestimación de los riesgos laborales sería el conocido sesgo de publicación, que determina que los estudios con resultados negativos (es decir, los que no encontrarían asociación entre las exposiciones laborales y las alteraciones de interés) tengan menos probabilidad de ser publicados, y viceversa. Desconocemos el impacto que este efecto pudiera tener sobre los estimadores utilizados en este trabajo.

En relación con la estimación de costes, en el presente estudio este aspecto no se ha abordado exhaustivamente, limitándonos a una aproximación a los costes más fácilmente cuantificables. En nuestros cálculos se han tenido en cuenta solamente los costes económicos por pérdida de productividad laboral, dejando sin consideración otros costes derivados de la mortalidad laboral (sanitarios, sociales, humanos, etc.). De hecho, los costes por pérdida de productividad estimados en este trabajo son conservadores, ya que se basan exclusivamente en los salarios, es decir, sólo tienen en cuenta los costes de productividad por la parte del trabajador. 
En definitiva, la valoración conjunta de la estrategia seguida en nuestro trabajo para estimar las muertes de origen laboral ocurridas en España y sus correspondientes costes por pérdidas de productividad, sugiere que es poco probable que el método aplicado sobrestime el verdadero impacto de la mortalidad laboral en nuestro país. Mientras el sistema de reconocimiento de la mortalidad por enfermedades laborales sea tan marcadamente insuficiente para cuantificar las dimensiones reales de la situación, estimaciones como las presentadas en este estudio pueden contribuir a poner de manifiesto la importancia de las condiciones de trabajo como determinantes de enfermedades y muertes evitables en España, y la inminente necesidad de sistemas de vigilancia y prevención adecuados para afrontar en toda su magnitud este problema principal para la salud pública.

\section{AGRADECIMIENTOS}

A la Cátedra Universidad-Empresa-Sindicato "Trabajo, Ambiente y Salud" por sus comentarios sobre el informe "Impacto de las enfermedades de origen laboral en España", del que proceden los datos presentados en este trabajo. A Julio López Bastida, por sus referencias y comentarios en relación con la valoración de los costes de las enfermedades. Este estudio se ha realizado con una ayuda del Ministerio de Trabajo y Asuntos Sociales (referencia FIPROS 2005-10).

\section{BIBLIOGRAFÍA}

1. Benavides FG, Castejón J, Gimeno D, Porta M, Mestres J, Simonet P. Certification of occupational diseases as common diseases in a primary health care setting. Am J Ind Med. 2005; 47:176-80.

2. Artieda L, Layana E, Lezaún M, Cipriain C. Diagnóstico de Salud Laboral en Navarra, 1997-1999. Pamplona: Instituto Navarro de Salud Laboral. Fondo de Publicaciones del Gobierno de Navarra; 2001 [citado 25 Oct 2006]. Disponible en: http://www.cfnavarra.es/insl/doc/Diagnostico99.pdf
3. Kogevinas M, Maqueda J, De la Orden V, Fernández F, Kauppinen T, Benavides FG. Exposición a carcinógenos laborales en España: aplicación de la base de datos CAREX. Arch Prev Riesgos Labor. 2000;3:153-9.

4. Kogevinas M, Rodríguez Suárez MM, Tardón A, Serra C. Cáncer laboral en España. Instituto Sindical de Trabajo, Ambiente y Salud, 2005 [citado 15 Nov 2006]. Disponible en: http://www.istas.ccoo.es/descargas/INFORMECANCER.pdf

5. García M. ¿Y para cuándo las enfermedades profesionales? Arch Prev Riesgos Labor. 1999;2:1-3.

6. Bofill J, Serra C, Benavides FG. La nueva lista de enfermedades profesionales, ¿qué novedades aporta respecto a la situación vigente hasta ahora? Arch Prev Riesgos Labor. 2007;2:69-71.

7. García M, Castañeda R. Enfermedades profesionales declaradas en hombres y mujeres en España en 2004. Rev Esp Salud Pública. 2006;80:361-75.

8. Driscoll T, Takala J, Steenland K, Corvalan C, Fingerhut M. Review of estimates of the global burden of injury and illness due to occupational exposures. Am J Ind Med. 2005;48:491-502.

9. Instituto Nacional de Seguridad e Higiene en el Trabajo. Anuario de estadísticas laborales y de asuntos sociales, 2006 [citado 7 Sep 2006]. Disponible en: http://www.mtas.es/insht/statistics/est_anuar.htm

10. Nurminen M, Karjalainen A. Epidemiologic estimate of the proportion of fatalities related to occupational factors in Finland. Scand J Work Environ Health. 2001;27:161-213.

11. Steenland K, Burnett C, Lalich N, Ward E, Hurrell J. Dying for work: the magnitude of US mortality from selected causes of death associated with occupation. Am J Ind Med. 2003;43:461-82.

12. García AM, Gadea R. Estimación de la mortalidad y morbilidad por enfermedades laborales en España. Arch Prev Riesgos Labor. 2004; 7:3-8.

13. Instituto Nacional de Estadística. Defunciones según la causa de muerte, 2004. [citado 7 Sep 2006]. Disponible en: http://www.ine.es/inebase

14. Instituto Nacional de Estadística. Indicadores demográficos básicos, 2004 [citado 7 Sep 2006]. Disponible en: http://www.ine.es/inebase

15. Instituto Nacional de Estadística. Encuesta Anual del Coste, 2004 [citado 15 Nov 2006]. Disponible en: http://www.ine.es/inebase 
16. Banco de España. Indicadores económicos, 2006 [citado 20 Nov 2006]. Disponible en: http://www.bde.es/infoest/indeco.htm

17. Tovar Jiménez J. Manual de Matemáticas Financieras [citado 13 Nov 2006]. Disponible en: http://www.matematicas-financieras.com/

18. Ministerio de Trabajo y Asuntos Sociales. Estadística de Accidentes de Trabajo. Año 2004 [citado 13 Nov 2006]. Disponible en: http://www.mtas.es/Estadisticas/EAT/eat04/index.htm

19. Instituto de Salud Carlos III. Mortalidad y Años Potenciales de Vida Perdidos, 2001 [citado 20 Nov 2006]. Disponible en: http://www.isciii.es/htdocs/ centros/epidemiologia/anexos/ww01_cap_ca.htm

20. Kraut A. Estimates of the extent of morbidity and mortality due to occupational diseases in Canada. Am J Ind Med. 1994;25:267-78.

21. Eurostat. Population and social conditions. Health and safety at work [citado 7 Sep 2006]. Disponible en: http://epp.eurostat.ec.europa.eu/
22. Riihimäki H, Kurppa K, Karjalainen A, Palo L, Jolanki R, Keskinen H, et al. Occupational diseases in Finland in 2002 New cases of occupational diseases reported to the Finnish Register of Occupational Diseases. Helsinki: Finnish Institute of Occupational Health; 2004.

23. Benavides FG, coordinador. Informe de Salud Laboral. España, 2006. Barcelona: Observatorio de Salud Laboral; 2007.

24. Artazcoz L, Borrell C, Cortès I, Escribà-Agüir V, Cascant L. Los determinantes de la salud en una perspectiva integradora de los enfoques de género, clase social y trabajo. En: Borrell C, Artazcoz L, coordinadoras. Investigación sobre género y salud. V Monografía de la Sociedad Española de Epidemiología [en prensa].

25. Rial E. Las prioridades de investigación sobre seguridad y salud en el trabajo: el desafío para una Europa de 27. Arch Prev Riesgos Labor. 2006;9:56-9. 